\title{
A New Technique in saving Fingerprint with low volume by using Chaos Game and Fractal Theory
}

\author{
Mahdi Jampour ${ }^{* 1}$ Member of IEEE and Senior Member of IACSIT; \\ Mohammad M. Javidi ${ }^{2}$, Adel Soleymani Nejad ${ }^{3}$, Maryam Ashourzadeh ${ }^{4}$, Mahdi Yaghoobi $^{5}$ \\ $(1,4)$ Kerman Institute of Higher Education (KIHE), Kerman, Iran \\ (2) Shahid Bahonar University of Kerman - Department of Computer Science, Kerman, Iran \\ (3) Islamic Azad University Branch of Zarand, Zarand, Iran \\ (5) Islamic Azad University, Mashhad Branch, Mashhad, Iran
}

\begin{abstract}
Fingerprint is one of the simplest and most reliable biometric features of human for identification. In this study by using fractal theory and by the assistance of Chaos Game a new fractal is made from fingerprint. While making the new fractal by using Chaos Game mechanism some parameters, which can be used in identification process, can be deciphered. For this purpose, a fractal is made for each fingerprint, we save 10 parameters for every fingerprint, which have necessary information for identity, as said before. So we save 10 decimal parameters with 0.02 accuracy instead of saving the picture of a fingerprint or some parts of it. Now we improve the great volume of fingerprint pictures by using this model which employs fractal for knowing the personality.
\end{abstract}

Keywords - Fractal Theory, Chaos Game, Fingerprint Storage, Fingerprint Compression.

\section{INTRODUCTION}

$\mathrm{T}$ hese days, identification is known as an issue which is so interrelated with human biometric features that signature, face, voice, iris, fingerprint, etc. in human are used for this purpose [1]. Undoubtedly, one of the simplest and most reliable identification methods based on human biometric features is fingerprint which has been in use for more than a hundred years in human identification and the reason can be its stability and unity. Identification based on fingerprint is considered as one of popular methods in identification of individuals and simplicity of the mechanism is the cause of this popularity. Sir Francis Galton and Sir Edward Henry were the first people who worked on identification based on fingerprint $[2,3]$. Galton focused his studies on fingerprint features and the results of his study led to definition and identification of some features in fingerprint and he called them Minutiae. Some of these features are shown in figure 1. Unlike him, Henry studied the general structure of fingerprint and the

\footnotetext{
* Corresponding author, Address: Computer and IT Department at Kerman Institute of Higher Education, Kerman, Iran. Tel: +98 915519 4189; Fax: +98 341212 2723. E-Mail Address: Jampour@ IEEE.org (Mahdi Jampour).
}

results of his studies led to classification of fingerprints into five categories; figure 2 shows this classification for fingerprints. Studies of these two were so much deep and clear that even after a hundred years they are used in researches. Issue of mechanical and automatic identification of fingerprint after extensive studies was authorized by FBI in 1969 and after that this task was given to National Institute of Standards and Technology (NIST). Some other works have been carried out on this subject, for instance we can point to what Maltoni has done for this purpose. He and his colleagues, relying on techniques based on Minutiae which Galton had introduced, worked on basic features of fingerprint and did the identification by the aid of these features [4]. In another work which was presented by Polikarpova, he analyzed fingerprint with fractal outlook. In this report fractal method is used for identification [5]. Also in an article which Karaki presented with the assistance of his colleagues in 2007, like Maltoni by analyzing Minutiae and improvement of popular methods he worked on identification based on fingerprint [6, 7 and 8].

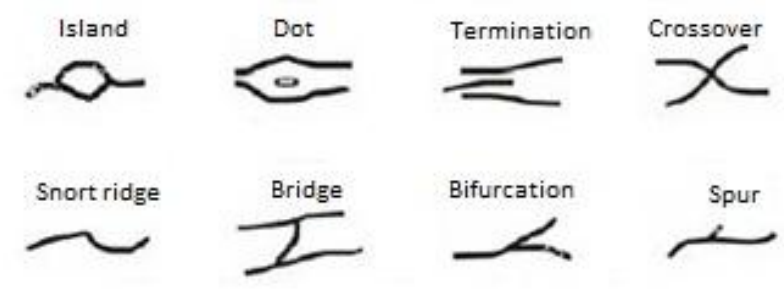

Fig. 1 the most common minutiae types.

\section{II.FINGERPRINT}

Ins and outs on tip of fingers are called fingerprints. Based on studies of Galton, there are some features in fingerprint which are different in various samples of fingerprints. These features are called Minutiae and averagely there are about 50 to 80 features in each fingerprint [9]. Core, delta, extension, ridge, cross, and island are some of these features. 

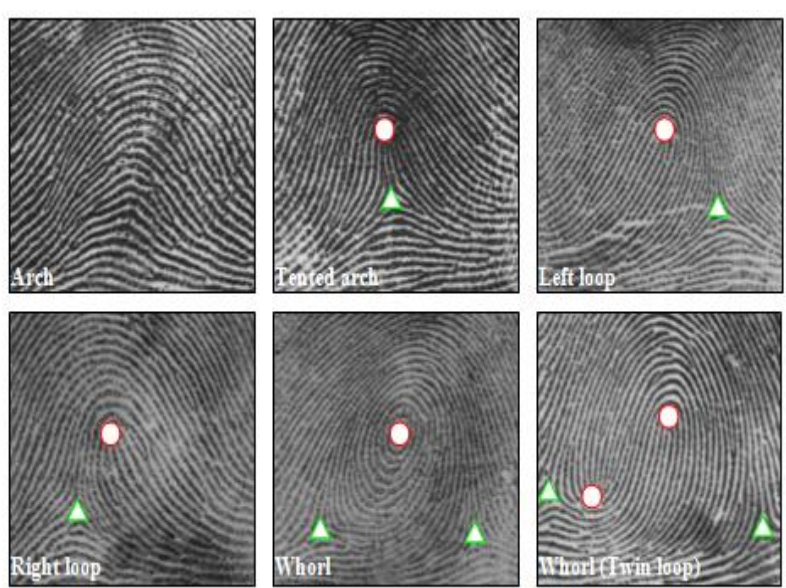

Fig. 2 Henry fingerprint classification, include: (a) Right loop, (b) Left loop, (c) Whorl, (d) Arch, (e) Tented Arch [4]

\section{A. Fingerprint Identification Methods}

Fingerprint identification methods are often divided into two main groups [10, 11 and 12]:

\section{1) Minutiae Based Algorithm (MBA) \\ 2) Pattern Based Algorithm (PBA)}

In the first type a fingerprint becomes ready for processing in a pre-processing stage and then by obtaining features of fingerprint it is compared to features in base with fingerprints. So it needs to save the minutiae's for using in mechanism. As determined, each fingerprint has 50-80 features. If 12 items of them adjust, it'll show fingerprint adjustment or that special case. Although more than 12 items protect for saving in base.

However, in pattern based method pattern of fingerprint sample is compared to other samples, level of conformity is calculated and on the basis of that a decision is made. In this method, protect the fingerprint or compressing form of it, is needed. In comparison to previous method, here we require more memory.

\section{FRACTAL THEORY}

The term fractal was introduced by Mandelbrot for the first time in 1975 [13, 14] and refers to geometry of objects which have the following features:

1. Self-similarity

2. Iterative Formation

3. Fractional Dimension

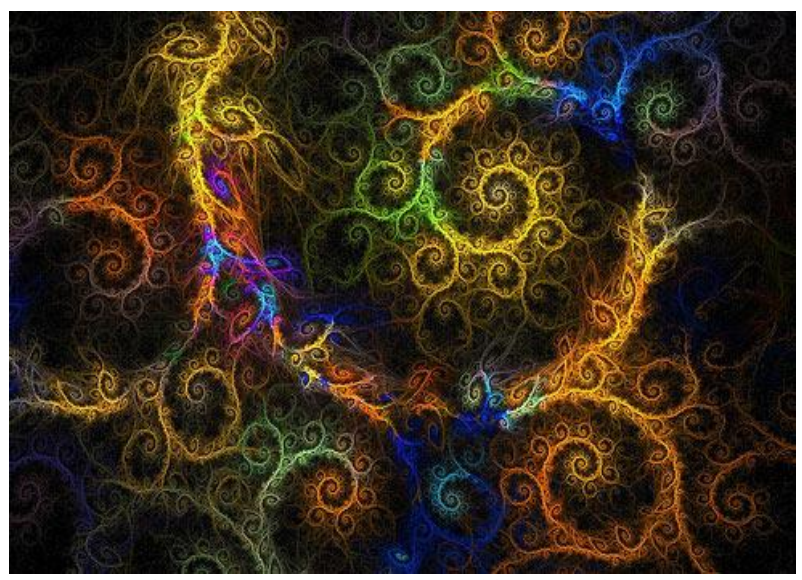

Fig. 3 I Sleep only (www.smashingmagazine.com)

It means that components of a fractal object are similar to whole of that object and are produced with a repetitive procedure and more importantly, if we calculate dimension of these objects, unlike objects like a line which has just one dimension or plate for which dimension is two, dimension of fractal objects is a decimal number[15]. In figure 3 a sample fractal is shown, also figure 4 shows another fractal which is known as Sierpinski Triangle and it has a dimension of 1.58 $[16,17]$

\section{A. Chaos Game Theory}

Chaos Game theory was presented by Barnsley in 1988 [18, 19]. This theory on the strength of Shannon Theorem is presented in a way that by using Random Walk mechanism and by the aid of a polygonal a fractal can be produced. This theory has two important points: first, by performing Chaos Game mechanism on a fractal a new fractal can be produced. Second and more important, while performing Chaos Game mechanism for producing the new fractal, besides properties of fractal some parameters can be deciphered which would be useful in identification process.

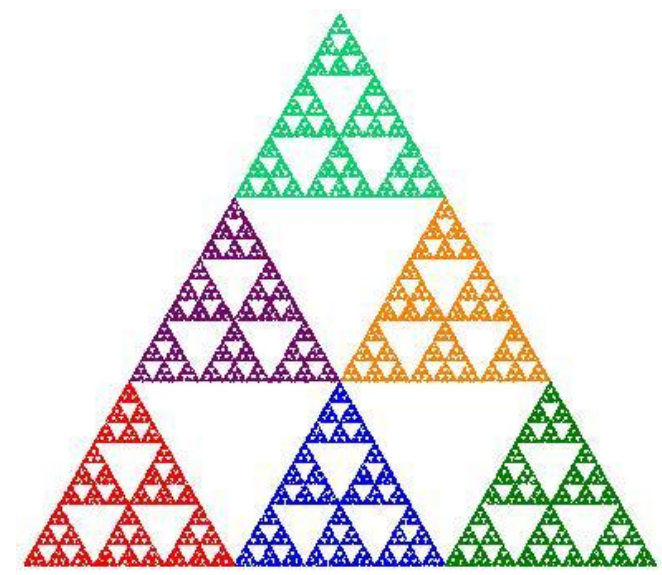

Fig. 4 Triangle, that its dimension is 1.58 


\section{B. Chaos Game Mechanism}

If we perform Chaos Game mechanism on a triangle, the procedure will be as the following: first, an accidental point is chosen. This point is the start point and it is not important in what situation it is. Then, in the second step an accidental number in the scope of [1...3] is chosen. If 1 is chosen it means summit $\mathrm{A}$, if 2 is chosen it means summit $\mathrm{B}$, and eventually if 3 is chosen it means summit $C$ in triangle. Then, from the present point we step forward half way toward chosen summit and draw a new point. Again, we make an accidental choice and repeat the same process for several times (for example 50000 times). This way a shape which is the very Sierpinski Triangle is drawn. In figure 5 four steps of Chaos Game mechanism are shown.

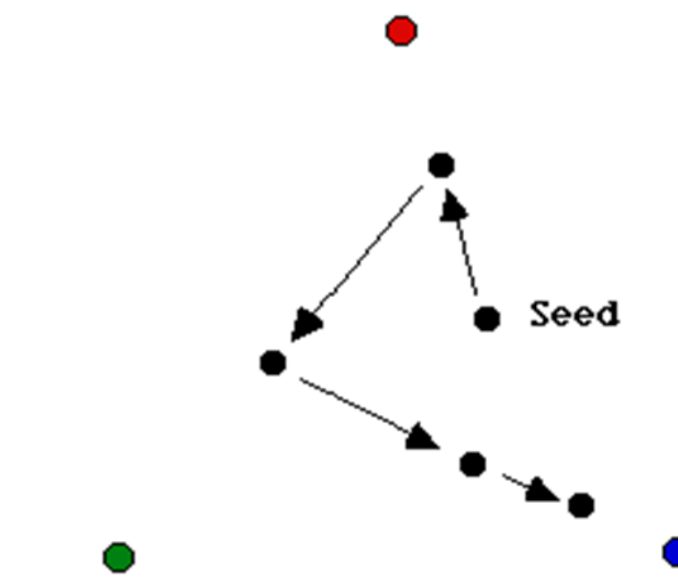

Fig. 5 Chaos Game mechanism on triangle that generate Sierpinski triangle

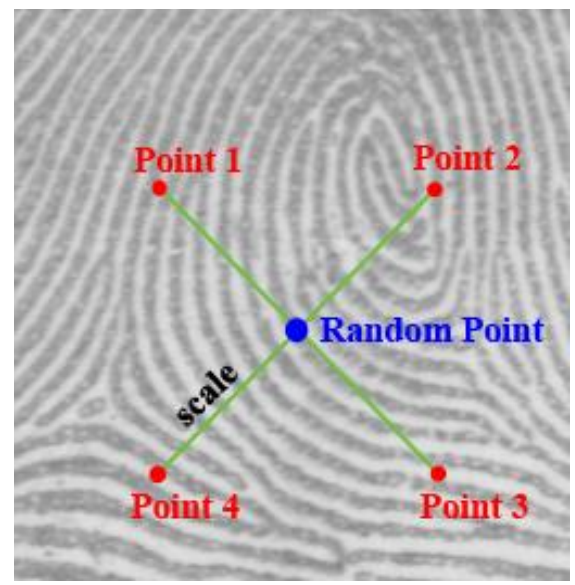

Fig. 6 Position of four assistant point's base on Random Point

\section{MAKING NEW FRACTAL BASED ON FINGERPRINT}

Fingerprint is a fractal and from fractal outlook it has fractal dimension parameter which is not sufficient for identification. As mentioned in 3-2, by the aid of Chaos Game a new fractal can be made and while making the new fractal more parameters can be obtained. As a result, based on a fingerprint and a pattern which will be pointed at afterwards the new fractal is made. Mechanism is in the way that a pixel of fingerprint is selected by chance. A point has 2 states: first, the pixel can be an in or zero or it might be an out or 1 . Both of these states cannot be sufficient for identification, so an accidental point is chosen and based on a new parameter which is called Scale we find four points around the aforementioned accidental point. If accidental point is $\mathrm{A}[\mathrm{i}, \mathrm{j}]$, four points will be:

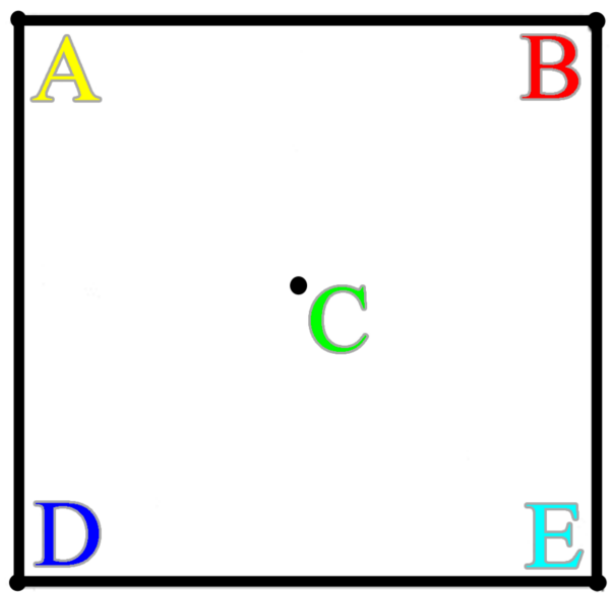

Fig. 7 five points that are mapped on five Categories in table1
Point $1=A[i-$ Scale, $j-$ Scale $]$
Point $2=A[i+$ Scale, $j-$ Scale $]$
Point $3=A[i+$ Scale, $j+$ Scale $]$
Point $4=A[i-$ Scale, $j+$ Scale $]$

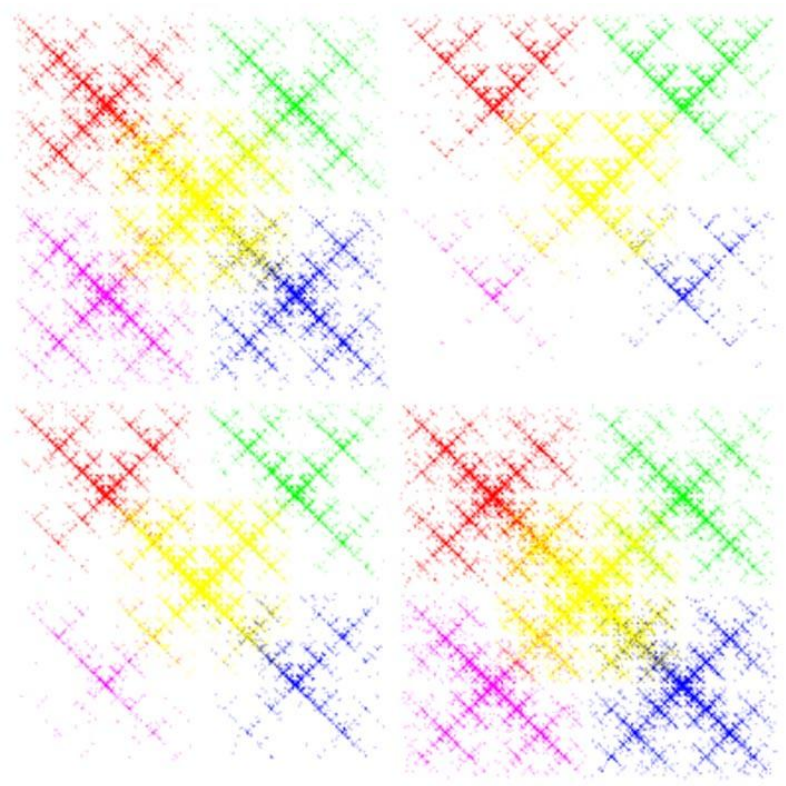

Fig. 8 four fractals that generated from four fingerprints 
These four points are shown in figure 6. Having these four points, 16 states are made which we divide into five distinct groups. First group is the state where pixels of all these four points are white, second group is the state where just one pixel from the mentioned points is black and all others are white. Third group is the state where the number of points with black pixels is two, fourth group is the state where the number of points with black pixels is three, and finally fifth group is a group where all four points have black pixels. Considering binary structure, these four points and creation of sixteen possible states, all of them and their assortment based on the mentioned classification are shown in table 1.16 created states in table 1 make five groups and for making a new fractal in Chaos Game process we use a square which is shown with five tips in figure 7. These five tips are named with alphabets to make the new fractal image which is gotten from the fingerprint. Result of fractal which is made by the above procedure for several samples of fingerprints is shown in figure 8 .

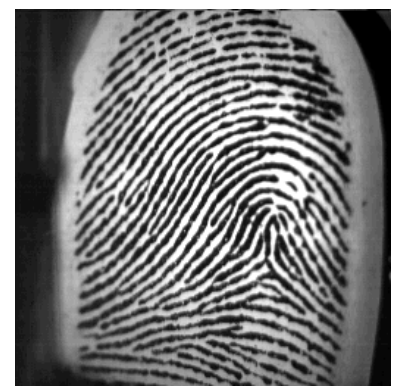

(a)

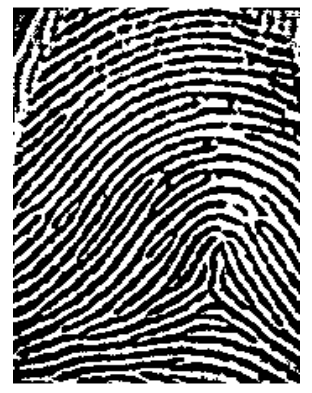

(b)
Fig. 9 a) before pre-processing, b) after pre-processing

Table 1 Categories modes may be relevant parts of the pixels

\begin{tabular}{|c|c|c|c|}
\hline Points $1-4$ & Category & Points $1-4$ & Category \\
\hline 0000 & 1 & 1000 & 2 \\
\hline 0001 & 2 & 1001 & 3 \\
\hline 0010 & 2 & 1010 & 3 \\
\hline 0011 & 3 & 1011 & 4 \\
\hline 0100 & 2 & 1100 & 3 \\
\hline 0101 & 3 & 1101 & 4 \\
\hline 0110 & 3 & 1110 & 4 \\
\hline 0111 & 4 & 1111 & 5 \\
\hline
\end{tabular}

\section{V.PARAMETER EXTRACTION}

As mentioned before, while making new fractal Chaos Game mechanism deciphers new parameters which can be useful in identification. In part four it was cited that regarding four defined points for pixels of those points, five states can be created which are classified into category1 to category5. Then, we repeat Chaos Game mechanism 100000 times and in each repetition by identifying four pixels of the points one of possible states is chosen and one unit is added to the counter of that category. This way, at the end of Chaos Game mechanism total of all five categories is equal to the number of repetitions, i.e. 100000. By dividing each of categories by 100000 they are standardized and we state them in the zero to one span. Therefore, for each fixed quantity for Scale parameter, which is the distance of points to the accidental point, five parameters are calculated. As five parameters with accuracy of (0.02) are not enough for identification, the above mentioned procedure is calculated for quantities of 10 and 15 for Scale.

As a result, we will have 10 parameters and considering the accuracy of 0.02 in the span of zero to one we will have 50 varieties. By having 10 parameters we will reach $50^{10}=9.76 \times 10^{16}$ varieties for fingerprints which guarantees unity of fingerprints. On the other hand, if we calculate Scale with different quantities for a fingerprint, we will have five independent spectrums and with the accuracy of 0.02 these spectrums are able to identify fingerprint based on the given mechanism. Figure 9 illustrates spectrums of parameters which are obtained from a sample fingerprint.

\section{IMPLEMENTATION}

The presented technique in this report has been carried out by MATLAB and has been experimented on 600 samples of fingerprint [20] from each of which there are four samples. A sample of used images is shown in figure 10.

$$
y=17.64 x+266
$$

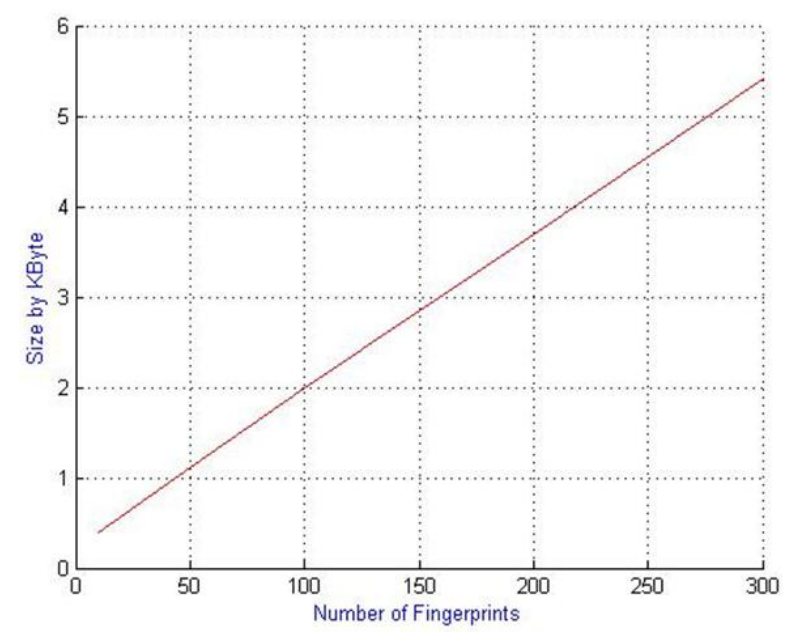

Fig. 10 linear growth rate of usage for storing Fingerprints

Results which are obtained show 100\% success for this technique. In another experiment 50 samples of fingerprints $20 \%$ of image of which were accidentally deleted were analyzed with the remaining parts and again $100 \%$ success was obtained and this shows stability of the presented method 
encountering deletion of some parts of image. Table 2 illustrates data related to time of searching in a base which includes 600 samples and also information related to time of deciphering and saving features of fingerprint in a database for some fingerprint samples. It must be noted that the experimenting system had a CPU Intel Celeron 2.60, $512 \mathrm{MB}$ RAM and Windows XP as its operating system.

Table 2 Amount of space for storing consumer features Fingerprint

\begin{tabular}{|cc|}
\hline Number of Fingerprints & Size on disk (KB) \\
\hline 10 & 0.40 \\
50 & 1.12 \\
100 & 1.98 \\
150 & 2.84 \\
200 & 3.70 \\
300 & 5.41 \\
\hline
\end{tabular}

\section{CONCLUSION}

Identification process by fingerprint based on popular methods such as Minutiae Based Algorithm and Pattern Based Algorithm, require fingerprint image information saving or feathers is derivation saving in minutiae style. The determined style in this article, fingerprint recognition will be done by using a new technique followed with fractal analyzing, only 10 parameters of fingerprint required to save, it causes less saving space compared with other methods. Also, in this article, it has been showed that by 10 parameters saving, we could find that specified fingerprint correctly in next search. The singularity of fingerprint has been determined. The result of used space for saving the fingerprint (KB) compared to the number of fingerprint has been showed in table 2 saving space increases by increasing in the number of fingerprint.

\section{REFERENCES}

[1] Jain, A.K. Flynn, P.J. Ross, A.A. Handbook of Biometrics, Springer, New York, (2008).

[2] Galton, F. Finger Prints, McMillian, London, (1892).

[3] Henry, E. Classification and Uses of Finger Prints, Routledge, London, (1900).

[4] Maltoni, D. Maio, D. Jain, A. Prabhakar, P. Handbook of Fingerprint Recognition, Springer, New York, (2003).

[5] Polikarpova, N. On the fractal features in fingerprint analysis. Proc. Int. Conf. Pattern Recognition, Austria, 3 (1996) 591.

[6] Karki, M.V. Sethu, S. A Novel Fingerprint Recognition System with Direction Angles Difference, Proc. Int. Conf. Computational Intelligence and Multimedia Applications, India, 2 (2007) 501.

[7] Jampour, M. Fingerprint Recognition with Fractal Theory. MSc. Thesis. Islam. Azad Univ. Mashhad. Iran. (2009).

[8] Jampour, M. Shojaei, H. Ashourzadeh, M. Yaghobi, M. Compressing of Fingerprint Images by Means of Fractals Feature. IEEE Int. Conf. ICMV'09 (2009) 18-22.

[9] Chikkerur, S. Pankanti, S. Jea, A. Ratha, N. Bolle, R. Fingerprint Representation Using Localized Texture Features, Proc. Int. Conf. Pattern Recognition, Hong Kong, 4 (2006) 521.
[10] Guo, H. A Hidden Markov Model Fingerprint Matching Approach, Proc. Int. Conf. Machine Learning and Cybernetics, China, 8 (2005) 5055.

[11] Zhou, J. Jinwei, G. A Model-Based Method for the Computation of Fingerprints' Orientation Field. IEEE Trans. Image Processing 13 (6) (2004) 821.

[12] Nanni, L. Lumini, A. Local binary patterns for a hybrid fingerprint matcher. Elsevier J. Pattern Recognition 41 (11) (2008) 3461.

[13] Mandelbrot, B. Fractals: Form, Chance, and Dimension. W. H. Freeman and Co, San Francisco, (1977).

[14] Mandelbrot, B. A multifractal walk down Wall Street. SCI AMERICAN INC, 280 (2) (1999) 70.

[15] Kigami, J. Analysis on fractals. Cambridge Univ. Press, Cambridge, (2001).

[16] Jampour, M. Yaghobi, M. Ashourzadeh, M. Fractal Images Compressing by Estimating the Closest Neighborhood with Assistance of Schema Theory. J. Com. Sci. 6(5) (2010) 591-596.

[17] Jampour $\mathrm{M}$, et al. Compressing images using fractal characteristic by estimating the nearest neighbor. IEEE Int. Conf. ITNG. (2009) 13191322.

[18] Barnsley, M.F. Fractals Everywhere. Academic Press, New York, (1988).

[19] Barnsley, M.F. SuperFractals. Cambridge University Press, New York, (2006).

[20] C.M. Brislawn, The FBI Fingerprint Image Compression Standard http://www.c3.lanl.gov/ brislawn/FBI/FBI.html

Mahdi Jampour is a researcher on Biometric Systems such as: Fingerprint, Iris, Face and etc. He got his B.S. in Computer Software from Shahid Bahonar University in 2006. He also got M.Sc. from Islamic Azad University, Mashhad branch in Artificial Intelligence (2009). He is a member of IEEE and a senior member of IACSIT. He wrote some papers in International Journals (ISI) including some conference papers which were published and presented in IEEE and ACM. He is editorial member of IJCTE and IJCA. He received a scholarship from Ministry of Science, Research and Technology (IRAN) to continue his studies towards Ph.D. He would like to do his research on Biometric, Chaos and Fractal, Fractal Image Compression, Genetic Algorithm, Schema Theory, Fuzzy Expert System and Fuzzy logic 